\title{
Patient and physician preferences for ulcerative colitis treatments in the United States
}

This article was published in the following Dove Press journal:

Clinical and Experimental Gastroenterology

\author{
Marco Boeri ${ }^{1}$ \\ Kelley Myers ${ }^{2}$ \\ Claire Ervin ${ }^{2}$ \\ Amy Marren ${ }^{3}$ \\ Marco DiBonaventura ${ }^{4}$ \\ Joseph C Cappelleri ${ }^{5}$ \\ Brett Hauber ${ }^{2}$ \\ David T Rubin ${ }^{6}$ \\ 'RTI Health Solutions, Health Preference \\ Assessment, Belfast, BT2 8LA, UK; ${ }^{2}$ RTI \\ Health Solutions, Health Preference \\ Assessment, Research Triangle Park, NC, \\ 27709, USA; ${ }^{3}$ Pfizer, Inflammation and \\ Immunology, Collegeville, PA, 19426, \\ USA; ${ }^{4}$ Pfizer, Health Economics and \\ Outcomes Research, New York, NY, \\ 10017, USA; ${ }^{5}$ Pfizer, Global Product \\ Development, Groton, CT, 06340, USA; \\ ${ }^{6}$ Department of Medicine, Inflammatory \\ Bowel Disease Center, University of \\ Chicago, Chicago, IL 60637, USA
}

Correspondence: Marco Boeri RTI Health Solutions, Forsyth House, Cromac Square Belfast BT2 8LA, UK Tel +44 I6I 4476016

Fax +4419195417222

Email mboeri@rti.org
Purpose: This study aimed to elicit patient and physician preferences for ulcerative colitis (UC) treatments in the United States (US).

Patients and methods: The following UC treatment attributes included in the discretechoice experiment (DCE) were identified during qualitative interviews with both patients and physicians: time to symptom improvement, chance of long-term symptom control, risks of serious infection and malignancy, mode and frequency of administration, and need for steroids. The DCE survey instruments were developed and administered to patients and physicians. A random-parameters logit model was used to estimate preference weights and conditional relative importance for these attributes.

Results: A total of 200 patients with moderate to severe UC (status determined using selfreported medication history) and 200 gastroenterologists completed the survey. Patients' average age was 42 years; most $(59 \%)$ were female. Patients considered symptom control 2.5 times as important as time to symptom improvement and 5-year risk of malignancy almost as important as long-term symptom control (relative importance, 0.79 vs 0.96 for long-term symptom control); they preferred oral to subcutaneous or intravenous administration (relative importance, 0.47 vs 0.11 and 0.18 , respectively). For physicians, symptom control was the most important attribute and was five times as important as the risk of malignancy.

Conclusion: Both patients and physicians considered long-term symptom control the most important attribute relative to others; however, risk of malignancy was of almost-equal importance to patients but not physicians. Differences between patients' and physicians' preferences highlight the need for improved communication about the relevant benefits and risks of different UC treatments to improve therapeutic decision-making.

Keywords: ulcerative colitis, discrete-choice experiments, maximum acceptable risk, patient preference, physician preference

\section{Introduction}

Ulcerative colitis (UC) is a chronic and debilitating inflammatory bowel disease (IBD), affecting more than half a million people in the United States (US). ${ }^{1}$ The disease is characterized by active periods during which patients experience a variety of symptoms ranging from mild to severe, such as abdominal pain, bloody diarrhea, fatigue and pale skin, poor appetite and weight loss, and loss of bowel movement control, followed by periods of remission. Although there is no generally accepted standard of care for moderate to severe UC, the past 10 years have seen considerable advances in management strategies for this condition. ${ }^{2}$ Recently released updated guidelines for the management of severe UC in adults have been published by the National Institute for Health and Care Excellence in the United Kingdom, ${ }^{3}$ 
and by the American College of Gastroenterology in the US. ${ }^{4}$ The main goal of treatment is to achieve and maintain remission, in turn improving patients' quality of life. The approach to treatment depends largely on the severity of the disease and whether a patient has become refractory to previous treatments. For patients with moderate to severe disease, the treatment pathway can include one or more medical treatments or may require surgery.

Currently, several medical treatments are available for patients with moderate to severe UC, including 5-aminosalicylates (5-ASAs), corticosteroids, immunosuppressants, and biologic therapies. Each of these treatments is characterized by different levels of efficacy, safety, and mode and frequency of administration; however, very little is known about how much value patients and physicians place on each of these attributes when making UC treatment decisions.

To date, few studies have examined patient and physician preferences for medical treatments for moderate to severe UC. Of the four stated-preference studies for UC treatments, two focused on comparing medical (nonsurgical) treatment and surgery in patients with moderate to severe UC, ${ }^{5,6}$ one explored IBD patients' willingness to accept medication risks to avoid future disease relapse, ${ }^{7}$ and the fourth estimated the differences in preferences and willingness to pay for 5-ASA treatments across four different countries and by levels of self-reported adherence in patients with mild to moderate UC. ${ }^{8}$ The results from these studies show that, in general, patients are willing to accept a certain level of risk of medication side effects for symptom relief and to avoid relapse, prefer the benefits of medical therapy over surgical therapy, and are generally averse to surgery as a UC treatment option.

Given the empirical evidence to support patient preferences for medical UC treatment options over surgery, ${ }^{5,6}$ it is important to understand how and to what degree treatment attributes influence preferences among medical treatments and whether these preferences differ from the patient and prescriber perspectives. No previous studies have explored and compared patient and physician preference for different characteristics of medications to treat moderate to severe UC. Therefore, the purpose of this study was two-fold: first, we aimed to independently identify the UC treatment attributes that patients and physicians consider most important when making treatment decisions and why, by conducting independent individual interviews with patients with UC and gastroenterologists; second, we aimed to elicit and quantify patient and physician preferences for the attributes of moderate to severe UC treatments that emerged from the qualitative interviews.

\section{Materials and methods}

\section{Study design}

To achieve our objectives, this study was conducted in two phases (Figure 1). During Phase I, we conducted individual interviews with patients with moderate to severe UC and gastroenterologists to identify the UC treatment attributes that patients and physicians consider most important and created a draft discrete-choice experiment (DCE) survey instrument to elicit patient and physician preferences for these attributes. During the second (quantitative) phase, a DCE survey instrument was pretested, finalized, and administered to patients and physicians to elicit their preferences and the relative importance of each of the treatment attributes included in the study.

Before starting the study, we determined criteria for including attributes: first, attributes needed to be relevant to patients and physicians when selecting their treatment across a series of alternatives; second, they needed to differentiate between available alternatives (as well as alternatives known to be under development by the study team or likely to be developed in the near future) in this indication. Phase I of the study involved in-depth qualitative interviews with both UC patients (face-to-face interviews, $\mathrm{N}=15$ ) and gastroenterologists (telephone interviews, $\mathrm{N}=16$ ). Participants were recruited by medical recruiters at a qualitative research firm. The screening criteria for the patient and physician participants are described below. Each interview was conducted by the same experienced interviewer, according to a semistructured interview guide. To elicit a comprehensive list of treatment attributes that influence patients' choice of UC treatments, patients were first asked about their experiences with UC and its treatments. Patients were then asked what they liked and did not like about current and previous treatments, as well as which factors would influence their decision to try a new treatment.

Similarly, each of the physician interviews was conducted using a semistructured interview guide (by the same experienced interviewer). Physicians were asked to describe their UC prescribing practices (what they prescribe the most/least and why) and their experiences with and opinions of UC treatments (including benefits and concerns) spanning 5-ASAs, corticosteroids, immunomodulators, and biologics. Physicians were also asked to describe the factors that would influence their decision to prescribe a new treatment or to switch a patient from one treatment to another. 


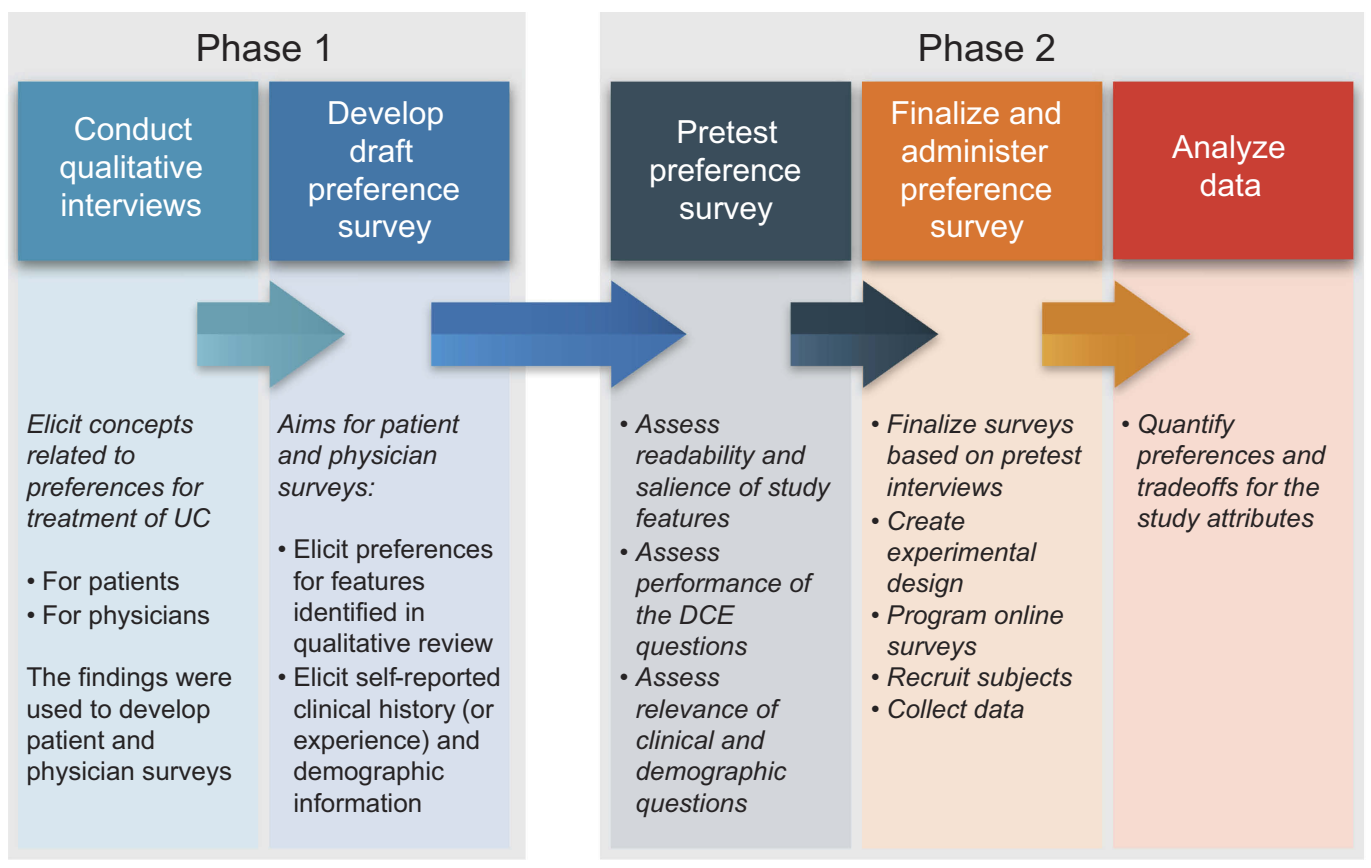

Figure I Study design.

A thematic analysis method ${ }^{9}$ was used to analyze the interview data. Important concepts and dominant trends were identified in each interview. These individual interview results were then compared across interviews to allow for the generation of themes or patterns in participants' responses. Frequencies and descriptive statistics were computed as applicable (see Supplementary Data). The same researcher led each interview as well as the analysis process, with input from the project team. Independent qualitative analyses were conducted with the interview data from each sample to account for the possibility that physicians and patients might be most influenced by different UC treatment attributes when making treatment choices. Those attributes described as the most important in the qualitative interviews by most patients and most physicians were considered for inclusion in the DCE.

The results indicated that the same concepts were most important and salient to both patients and physicians when selecting a UC treatment. Therefore, the DCE survey instrument was developed including an identical set of attributes for both samples following good research practices. ${ }^{10}$ After reviewing the results from the qualitative interviews as well as the study objectives, the study team identified the following seven attributes: (1) time until symptoms improve, (2) probability that UC symptoms are under control after 1 year, (3) annual risk of serious infection, (4) 5-year risk of malignancy, (5) mode of administration, (6) dosing schedule, and (7) need for occasional use of steroids (Table 1). Although the latter did not emerge clearly from the interviews, the qualitative research revealed that patients were concerned about side effects associated with steroid use and that physicians preferred a treatment strategy that would avoid prolonged or repeated steroid use. Therefore, this attribute was considered important and relevant for the DCE and was included. On the other hand, although cost emerged as important for the majority of patients and physicians, the study team decided not to include it to avoid complicating the survey instrument since the objective of the study was to evaluate the characteristics of the treatments themselves and not any factors of the health care system and we did not aim to explore either sensitivity to co-payments or willingness to pay as a monetary value to patients (it is unlikely that patients will pay the actual cost of the treatment. Including a cost as an attribute other than a nominal co-payment requires effort to get respondents to take the cost attribute seriously. This is not uncommon, but it is typically reserved for those studies in which estimating willingness to pay is a central objective).

In Phase II of the study, the DCE survey instrument developed in Phase I was pretested, finalized, and administered to elicit preferences for the attributes selected in Phase I. In the DCE exercise, respondents chose between hypothetical UC treatment profiles defined as combinations of attribute levels from Table 1. The pattern of 


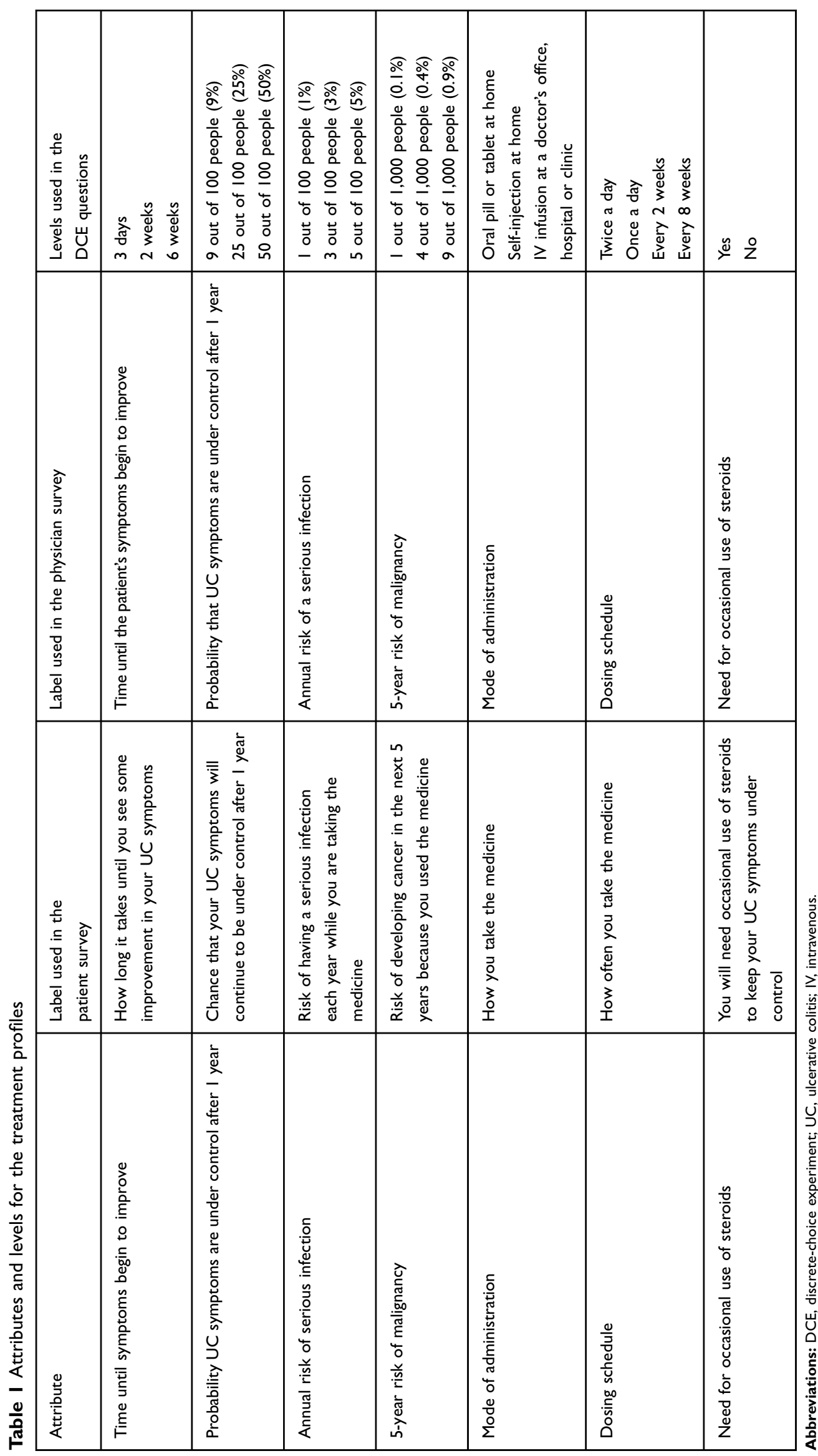


responses to a series of DCE questions reveals the tradeoffs respondents would be willing to make among treatment attributes and can be used to estimate the conditional relative importance of each attribute conditional on the range of the levels of that attribute. DCEs have been widely employed to quantify patients' and physicians' preferences and the trade-offs they are willing to accept between the benefits and risks of treatments. ${ }^{11-13}$

\section{Study populations}

The study populations included patients with moderate to severe UC and gastroenterologists who currently treat patients with moderate to severe UC. All respondents resided in the US at the time of the survey, were 18 years of age or older, and were able to read and understand English to provide informed consent and complete the survey. To be eligible for the study, patients had to have a self-reported physician diagnosis of moderate to severe UC (where a disease severity of moderate to severe was based on medication history), have no selfreported diagnosis of irritable bowel syndrome or Crohn's disease, and have taken a prescription medicine other than corticosteroids and 5-ASAs to treat their UC. Physicians had to be board-certified (or eligible) gastroenterologists who were currently treating at least 10 patients with moderate to severe UC each month. Participants were invited by Survey Sampling International (SSI; Shelton, Connecticut) to be screened for eligibility for the survey. Patients were invited through the SSI web panel in the US, and physicians were invited through an online blend of SSI and partner physician panels.

\section{Survey instruments}

The draft survey instruments developed in Phase I were pretested separately with convenience samples of 15 patients and 10 physicians. The patient survey instrument was pretested during 15 in-person interviews in Charlotte, North Carolina, whereas the physician survey instrument was pretested during 10 telephone interviews. The interviews confirmed that the attributes included in the draft survey were relevant to respondents, that no important attributes were omitted, that the survey length was appropriate, and that respondents understood and were willing to trade-off among treatment attributes.

In the choice questions presented to both patients and physicians in the final DCE survey instruments, each hypothetical UC treatment was defined by the following seven attributes: (1) time until symptoms improve, (2) probability that UC symptoms are under control after 1 year, (3) annual risk of serious infection,
(4) 5-year risk of malignancy, (5) mode of administration, (6) dosing schedule, and (7) need for occasional use of steroids (Table 1).

The hypothetical UC treatment options in the choice questions were constructed using an experimental design, such that each question required that respondents make trade-offs among the levels of treatment attributes and that such tradeoffs varied systematically across questions. We used Sawtooth Software to generate a D-optimal main-effects experimental design. ${ }^{14-16}$ Since the mode of administration and dosing schedule could not vary entirely independently, the design was constrained to exclude specific combinations, such as subcutaneous injection twice a day and intravenous infusion once or twice a day. The two attributes were therefore designed as though they were one attribute (dosing and schedule of administration) with nine levels. The experimental design included 48 choice questions, each with two hypothetical treatment profiles (same for patients and physicians). Questions were randomly ordered to mitigate order effects. In the patient sample, the design was split into four blocks of 12 choice questions. Patients were randomly assigned to one block of 12 choice questions. In the physician sample, the design was split into six blocks of eight choice questions. Physicians were assigned to three blocks (one for each of the three hypothetical patient profiles described below).

\section{Patient survey}

Patient respondents were asked to report disease and treatment experience and demographic characteristics. Patients were then presented the following scenario:

Suppose your doctor tells you that you need to start a new UC medicine because your current treatment is no longer working. The doctor says there are two medicines you can get, Medicine A or Medicine B. Both medicines will control your UC symptoms, but they will differ in other ways.

In the next 12 questions, we will show you different pairs of hypothetical UC medicines that your doctor might offer. For each pair of medicines, please select the medicine that you would choose if they were only two options available.

Suppose that all of the medicines would be available to you at no additional out-of-pocket cost, and they would all cost the same as your current treatment. The medicine you choose would replace any other UC medicines you might be taking now. 


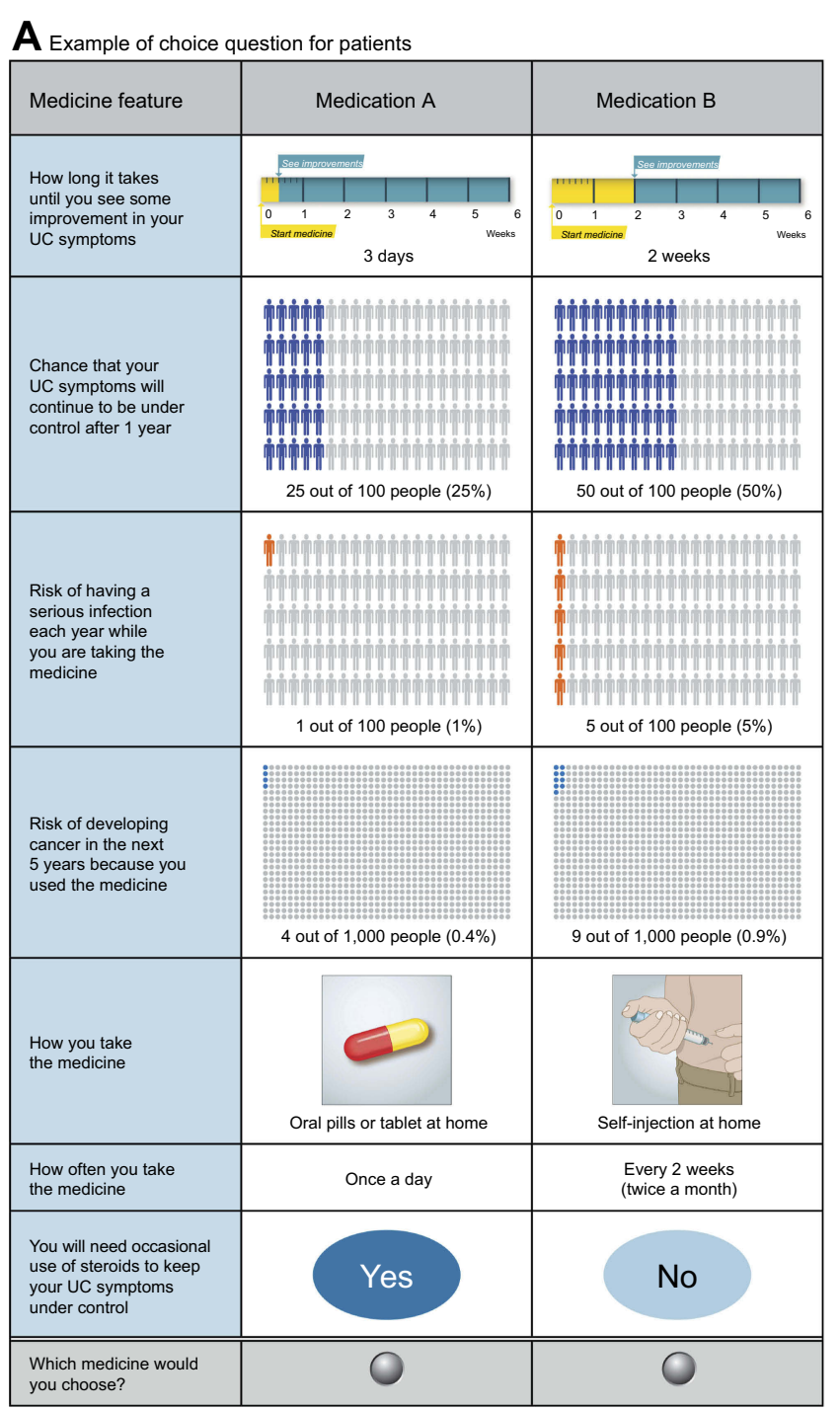

Figure 2 Example choice question.

Abbreviation: UC, ulcerative colitis.

Figure 2A presents an example of a choice question included in the patient survey instrument.

\section{Physician survey}

Physicians were asked to indicate, in a series of eight choice questions, which UC treatment they would recommend for each of three hypothetical patients, each with moderate to severe UC who had has previously failed on both immunomodulators and 5-ASAs, further described as follows:

- Patient 1: a young, single patient who works full time, lives in a suburban area, and has no children.

- Patient 2: a patient who is in his or her late $30 \mathrm{~s}$, is single with children, works part time, and lives in a rural area.
B Example of choice question for physicians

\begin{tabular}{|c|c|c|}
\hline Treatment feature & Treatment A & Treatment B \\
\hline & 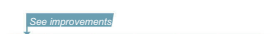 & 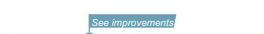 \\
\hline $\begin{array}{l}\text { Time until the } \\
\text { patient's symptoms } \\
\text { begin to improve }\end{array}$ & 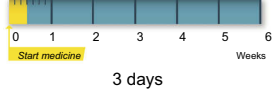 & 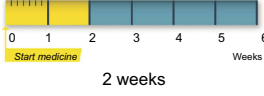 \\
\hline $\begin{array}{l}\text { Probability that } \\
\text { uC symptoms are } \\
\text { under control after } \\
1 \text { year }\end{array}$ & 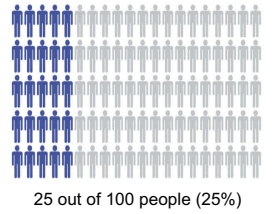 & 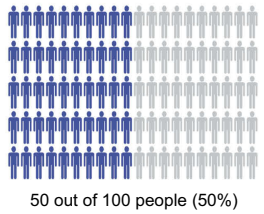 \\
\hline $\begin{array}{l}\text { Annual risk of a } \\
\text { serious infection }\end{array}$ & 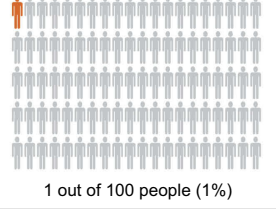 & $\begin{array}{l}\text { Pon } \\
5 \text { out of } 100 \text { people }(5 \%)\end{array}$ \\
\hline $\begin{array}{l}\text { 5-year risk of } \\
\text { malignancy }\end{array}$ & $\begin{array}{l}\text { Dom } \\
\text { D. }\end{array}$ & 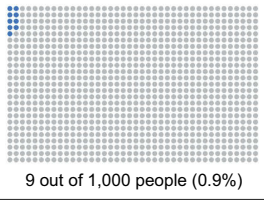 \\
\hline $\begin{array}{l}\text { Mode of } \\
\text { administration }\end{array}$ & & \\
\hline & Oral pills or tablet at home & Self-injection at home \\
\hline Dosing schedule & Once a day & Every 2 weeks \\
\hline $\begin{array}{l}\text { Need for } \\
\text { occasional use } \\
\text { of steroids }\end{array}$ & Yes & No \\
\hline $\begin{array}{l}\text { Which treatment } \\
\text { would dou prescribe } \\
\text { for this patient? }\end{array}$ & 0 & 0 \\
\hline
\end{tabular}

- Patient 3: an older patient who lives alone in a suburban area and is retired.

Half of the physician sample was presented with female patient profiles and half was presented with male patient profiles, yielding a total of six profiles.

Figure 2B presents an example of a choice question included in the physician survey instruments.

\section{Statistical analyses}

Choice data were analyzed using a random-parameters logit (RPL) model. The RPL model relates treatment choices from each respondent to the attribute levels of each treatment profile in the choice questions. The RPL model mitigates potential estimation bias in the mean preference-weight estimates that 
may arise due to unobserved preference heterogeneity among respondents by estimating a distribution around each mean preference parameter. ${ }^{17,18}$ In all RPL models, the random parameters corresponding to main effects were assumed to be normally distributed. We estimated an RPL model in which no attributes were interacted. In the final model, all independent variables were effects coded for each attribute so that the mean effect for each attribute was normalized at zero. Models for the patient and physician samples were estimated separately.

We tested whether physician preferences varied systematically among patient profiles and between patient genders. Both a Wald test and the test on pooled DCE data sets proposed by Swait and Louviere ${ }^{19}$ were used for this analysis. There was no evidence of systematically statistically significant differences in preferences across hypothetical patient profiles or between male and female profiles; consequently, the data for all profiles were pooled in the analysis of the physician data.

Following the recently published good research practices for the analysis of DCE data, ${ }^{20}$ we analyzed the pattern of response to the hypothetical choice questions to estimate a relative preference weight for each attribute level included in the study. These results were used to estimate the conditional relative importance of each attribute by calculating the difference between the estimated preference weights for the mostand the least-preferred levels of that attribute. Conditional relative importance is the importance of an attribute relative to all other attributes included in the study, conditional on the range of levels of each attribute. To compare the conditional relative importance between the physician and patient samples, we set the relative importance for the attribute with the highest conditional relative importance to 10 for both samples.

Finally, the RPL results were used to calculate the respondents' maximum acceptable percentage-point increase in the risks of serious infection and malignancy that patients and physicians would accept for each of the changes in each of the remaining attributes: time until symptoms begin to improve, the probability that UC symptoms would be under control after 1 year, need for occasional use of steroids, changes in frequency for the same mode of administration, and changes in mode of administration for each frequency. This was computed as the negative ratio between the difference in preferences (utility) for the change in two levels of an attribute and the disutility of a unit change in the risk of serious infection and malignancy. Since the risk was coded as categorical, the disutility between two levels was modeled assuming that preferences were linear between each pair of effects-coded risk levels included in the survey instrument.

\section{Results}

\section{Respondent characteristics}

A total of 3,873 patients from the SSI web panel in the US were screened for eligibility for the survey, of whom 709 responded by accessing the survey. Of those who responded, 278 were eligible to take the survey, 264 consented to participate, and 200 patients completed the survey. The final sample of 200 patient respondents was $59.0 \%$ female, with a mean age of 42.1 years. Most respondents had a 4-year college degree or higher $(55.5 \%)$ and were employed full time $(61.0 \%)$ (Table 2$)$.

Approximately 2,630 physicians from the SSI web panel and partner panels were notified of potential study eligibility, of whom 784 accessed the survey link, 213 were eligible to take the survey, 212 (99.5\%) consented to participate, and 200 physicians completed the survey. The final sample of 200 physician respondents was $73.0 \%$ male, with a mean age of 48.7 years. Most of the physicians included in the final sample worked in an officebased private practice $(60.0 \%)$ and treated more than 15 UC patients per month (70\%) (Table 3 ).

\section{Preference weights}

The estimated preference weights for patients and physicians are presented in Figure 3.

For patients, we found that 2 weeks until symptoms begin to improve was preferred to 6 weeks, but not to 3 days. However, there is no statistical difference between 2 weeks and 3 days. In addition, we found that a higher probability that symptoms would be under control after 1 year and a lower risk of both adverse events were preferred to a lower probability that symptoms would be under control after 1 year and to a higher risk of both adverse events.

When considering the mode of administration independent of the dosing schedule, patient respondents preferred pills to subcutaneous injections or intravenous infusions. However, the difference in preference weights between subcutaneous injections or intravenous infusions was not statistically significant, potentially indicating that respondents were, on average, indifferent between these two modes of administration. When both mode of administration and dosing schedule were considered, a more frequent dosing schedule was preferred to a less frequent dosing schedule for pills (eg, a pill twice a day was preferred to a pill every 8 weeks). However, these differences were not statistically significant within each mode of administration. Finally, whether the treatment required the 
Table 2 Respondent characteristics

\begin{tabular}{|c|c|}
\hline Survey question & Responses, n (\%) \\
\hline \multicolumn{2}{|l|}{ Patients (N=200) } \\
\hline Mean (SD) age, years & $42.1(14.68)$ \\
\hline \multicolumn{2}{|l|}{ Gender } \\
\hline Female & I I 8 (59.0) \\
\hline Male & $82(4 \mid .0)$ \\
\hline \multicolumn{2}{|l|}{ Marital status } \\
\hline Single/never married & $68(34.0)$ \\
\hline Married/living as married/civil partnership & II $3(56.5)$ \\
\hline Other & $19(9.5)$ \\
\hline \multicolumn{2}{|l|}{ Highest level of education } \\
\hline Some high school & $2(1.0)$ \\
\hline High school or equivalent (eg, GED) & $21(10.5)$ \\
\hline Some college but no degree & $32(16.0)$ \\
\hline Technical school & $9(4.5)$ \\
\hline Associate's degree (2-year college degree) & $25(12.5)$ \\
\hline 4-year college degree (eg, BA, BS) & $71(35.5)$ \\
\hline Some graduate school but no degree & $3(1.5)$ \\
\hline Graduate or professional degree & $37(18.5)$ \\
\hline \multicolumn{2}{|l|}{ Employment status } \\
\hline Employed full time & $122(6 \mid .0)$ \\
\hline Employed part time & $22(11.0)$ \\
\hline Self-employed & $10(5.0)$ \\
\hline Retired & $16(8.0)$ \\
\hline Other & $30(15.0)$ \\
\hline \multicolumn{2}{|l|}{ Type of health insurance ${ }^{a}$} \\
\hline I do not have health insurance & $2(1.0)$ \\
\hline Private insurance that I pay for myself & $45(22.5)$ \\
\hline Private insurance that my employer or my spouse's employer pays all or part of & $98(49.0)$ \\
\hline Medicaid & $22(11.0)$ \\
\hline Medicare & $40(20.0)$ \\
\hline Veteran Health Insurance & $3(1.5)$ \\
\hline Other & $5(2.5)$ \\
\hline Mean (SD) age at UC diagnosis, years & $33.0(14.45)$ \\
\hline \multicolumn{2}{|l|}{ Prescription medications ever taken for $\mathrm{UC}^{\mathrm{a}}$} \\
\hline Immunosuppressants & $106(53.0)$ \\
\hline Biologics & $98(49.0)$ \\
\hline 5-aminosalicylates (5-ASAs) & $81(40.5)$ \\
\hline Corticosteroid & $103(51.5)$ \\
\hline Another prescription medication for UC & $28(14.0)$ \\
\hline \multicolumn{2}{|l|}{ Experience with modes of administration for UC medication ${ }^{\mathrm{a}}$} \\
\hline By mouth (oral pills or tablets) & $178(89.0)$ \\
\hline By injection (either at home or in your doctor's office) & $81(40.5)$ \\
\hline By intravenous (IV) infusion & $52(26.0)$ \\
\hline Suppository (capsule inserted in your rectum) & $43(21.5)$ \\
\hline Others & $4(2.0)$ \\
\hline Don't know or not sure & I (0.5) \\
\hline
\end{tabular}

(Continued) 
Table 2 (Continued).

\begin{tabular}{|c|c|}
\hline Survey question & Responses, n (\%) \\
\hline \multicolumn{2}{|l|}{ Physicians (N=200) } \\
\hline Mean (SD) age, years ${ }^{\mathrm{b}}$ & $48.7(10.39)$ \\
\hline \multicolumn{2}{|l|}{ Gender } \\
\hline Female & $48(24.0)$ \\
\hline Male & $146(73.0)$ \\
\hline Prefer not to say & $6(3.0)$ \\
\hline \multicolumn{2}{|l|}{ Years in practice } \\
\hline 6 years or less & $42(21.0)$ \\
\hline $7-15$ years & $67(33.5)$ \\
\hline More than 15 years & $91(45.5)$ \\
\hline \multicolumn{2}{|l|}{ Practice type ${ }^{a}$} \\
\hline Office-based private practice & $120(60.0)$ \\
\hline Hospital-based private practice & $41(20.5)$ \\
\hline Academic-based practice & $46(23.0)$ \\
\hline Has infusion chair or suite in practice & I6I (80.5) \\
\hline \multicolumn{2}{|c|}{ Number of patients with UC seen each month } \\
\hline $10-15$ patients & $60(30.0)$ \\
\hline 16-25 patients & $61(30.5)$ \\
\hline More than 25 patients & $79(39.5)$ \\
\hline \multicolumn{2}{|c|}{ Years of experience treating patients with moderate to severe UC } \\
\hline $\mathrm{I}-5$ years & $15(7.5)$ \\
\hline $6-10$ years & $48(24.0)$ \\
\hline $\mathrm{II}-15$ years & $42(21.0)$ \\
\hline More than 15 years & $95(47.5)$ \\
\hline \multicolumn{2}{|c|}{ Percentage of patients with moderate to severe UC currently using biologics } \\
\hline $0-20 \%$ & $20(10.0)$ \\
\hline $21-45 \%$ & $5 I(25.5)$ \\
\hline $46-75 \%$ & $71(35.5)$ \\
\hline More than $75 \%$ & $58(29.0)$ \\
\hline
\end{tabular}

Notes: ${ }^{a}$ Respondents had the option to select more than one response category. For this reason, the percentages may sum to more than $100 \%$. ${ }^{b}$ One respondent did not provide an answer to this question.

Abbreviations: GED, General Education Development; SD, standard deviation; UC, ulcerative colitis.

occasional use of steroids or not did not significantly impact patients' preferences.

For physicians, we found that preferences for the clinical outcomes included in the DCE were ordered as expected: faster onset of action was preferred to longer time until symptoms begin to improve, higher probabilities that symptoms would be under control after 1 year were preferred to lower probabilities, and lower levels of annual risk of serious infection and 5-year risk of malignancy were preferred to higher levels of these risks. When considering mode of administration and dosing schedule, physicians preferred pills to subcutaneous injections or intravenous infusions independently from the dosing schedule, and they preferred a less frequent dosing schedule to a more frequent dosing schedule for both subcutaneous injection and intravenous infusions.

\section{Comparing patients and physicians: conditional relative importance}

The utility variation caused by a change in the levels of each attribute is represented by the vertical distance between the preference weights for any two levels of that attribute in both Figure 3A (for patients) and Figure 3B (for physicians). The vertical distance between the most-preferred and leastpreferred levels of an attribute is also a measure of conditional relative importance of that attribute - the importance of an 


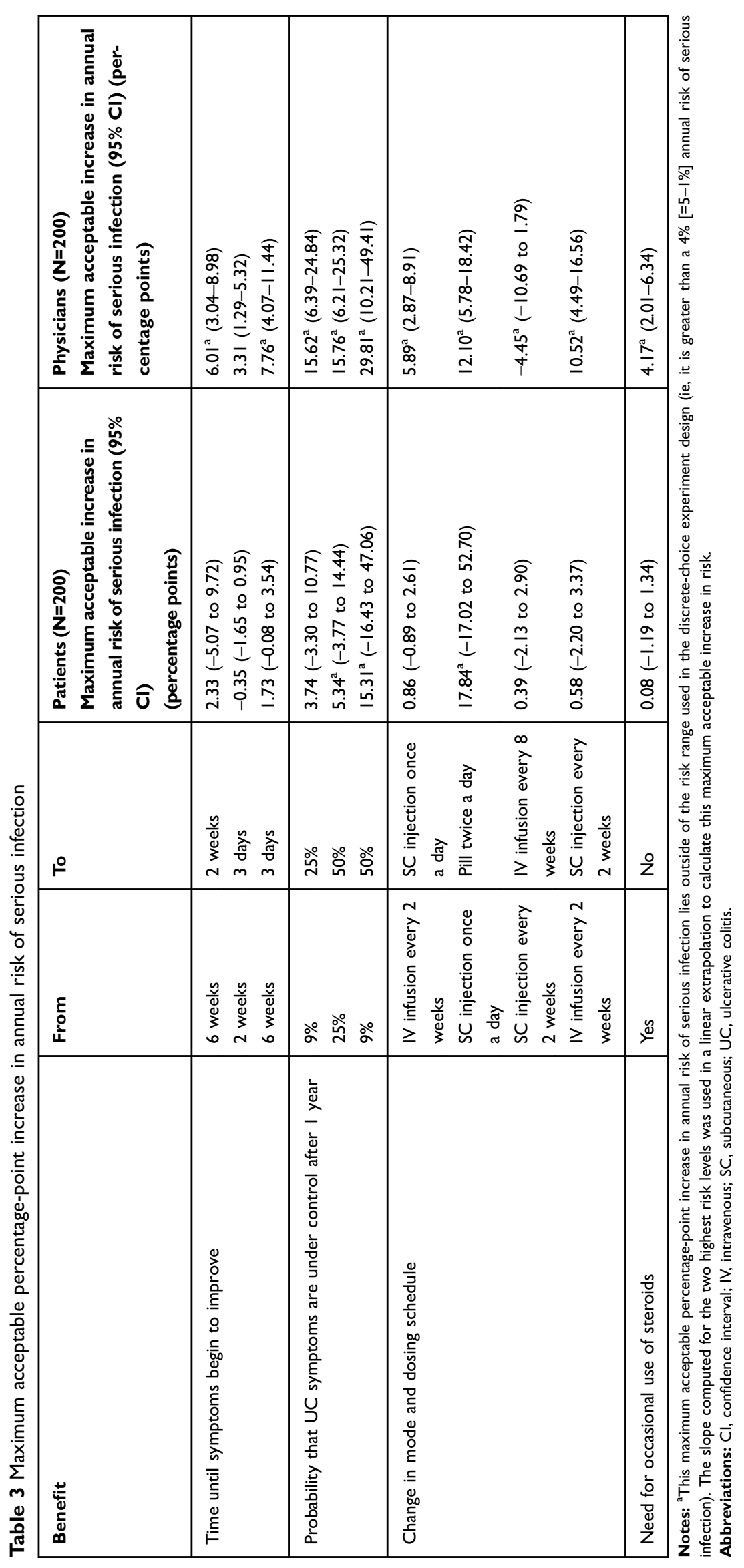



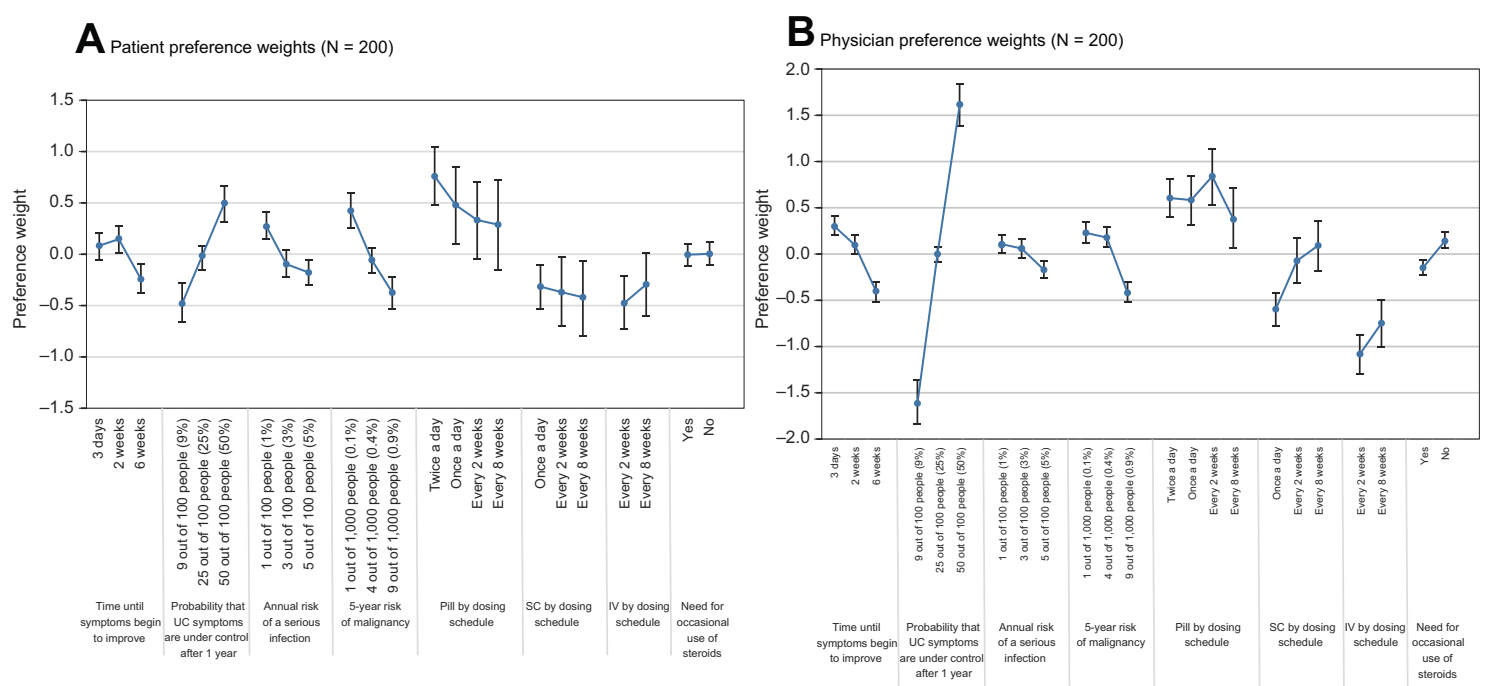

Figure 3 Preference weights. Notes: This graph presents the preference weights placed on the attribute levels represented on the horizontal axis. Vertical distance between the preferences for various levels of the same attribute represents the weight placed on a specific relative change in that attribute. Calculated mean preference estimates for each value can be compared within each attribute and across different attributes. The vertical bars surrounding each mean preference weight denote the $95 \%$ confidence interval about the point estimate (computed by delta method).

Abbreviations: IV, intravenous; SC, subcutaneous; UC, ulcerative colitis.

attribute relative to the other attributes in the study given the range of levels of that attribute and other attributes. Larger differences between preference weights indicate that respondents viewed the change as relatively more important. To facilitate the comparison between patient and physician preferences, Figure 4 reports conditional relative importance of each UC treatment attributes included in this study for both patients and physicians, relative to the probability that UC symptoms would be under control after 1 year-the attribute with the highest conditional relative importance. In Figure 4, the conditional relative importance of the most important attribute is set to 10 , and the conditional importance of each of the other attributes is scaled relative to it.

Statistical conclusions about differences (or lack thereof) in the conditional relative importance estimates shown in Figure 4 cannot be drawn by comparing the two models directly. Differences between patients and physicians can be inferred only on the basis of the strong assumption that the most important attribute (the probability that UC symptoms would be under control after 1 year) is equally important to both patients and physicians. Once this assumption is accepted, we can use standardized values of conditional relative attribute importance to compare patient and physician preferences. For example, Figure 4 shows that while patients considered symptom control 2.5 times as important as time to symptom improvement, and 5-year risk of malignancy approximately $80 \%$ as important as long-term symptom

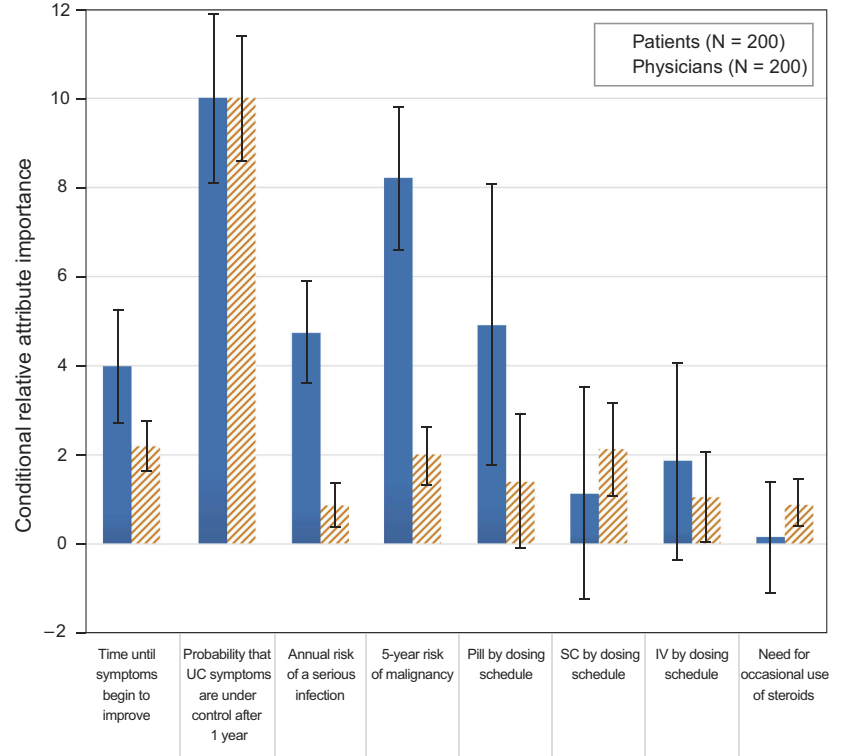

Figure 4 Conditional relative importance for patients and physicians.

Notes: This graph plots the conditional relative importance of each attribute, calculated as the difference between the most-preferred and least-preferred level. The importance of each attribute for both patients and physicians is relative to the probability that UC symptoms would be under control after I year and is conditional on the levels of the attributes included in the survey. The black vertical bars surrounding each mean preference weight denote the $95 \%$ confidence interval about the point estimate. The conditional relative importance for each mode of administration (ie, pill, subcutaneous injection, and intravenous infusion) is reported over the range of frequency levels associated with it.

Abbreviations: IV, intravenous; SC, subcutaneous; UC, ulcerative colitis.

control (the conditional relative attribute importance is 0.79 and 0.96 respectively), for physicians the conditional relative importance of the 5 -year risk of malignancy is only $20 \%$ as 
important as the probability that UC symptoms are under control after 1 year. This comparison suggests that patients are more averse to risk than physicians.

\section{Maximum acceptable increase in risk of adverse events}

Table 3 presents results obtained by computing the maximum acceptable percentage-point increase in the annual risk of serious infection to obtain an increase in efficacy, to change the mode and dosing schedule, and to avoid the need for the occasional use of steroids. Most of the other trade-offs included in Table 4 were not statistically significantly different from zero for patients or were above the range of risk level included in the DCE experimental design.

In general, the maximum acceptable increase in risk of annual serious infection was not statistically significantly different from zero for patients. This is mainly due to the wide confidence interval generated by the calculation. Because all confidence intervals for the estimates of maximum acceptable increase in the risk of serious infection based on our sample include zero, there is no statistical evidence to suggest that respondents were willing to accept any increase in the annual risk of serious infection to increase efficacy, avoid the occasional use of steroids, or change the mode of administration and dosing schedule.

For physicians, the maximum acceptable increases in risk of annual serious infection shown are statistically significantly different from zero, indicating that physicians in our sample were generally willing to accept increases in the annual risk of serious infection to increase efficacy, to avoid the occasional use of steroids, or to change the mode of administration and the dosing schedule for hypothetical patients.

Table 4 presents the maximum increase in the 5-year risk of malignancy acceptable to obtain an increase in efficacy, to change mode and dosing schedule, and to avoid the need for the occasional use of steroids. Most of the other trade-offs included in Table 4 were not statistically significantly different from zero for patients or were above the range of risk level included in the DCE experimental design.

Patients were willing to accept approximately twotenths of a percentage-point increase $(0.2 \%)$ in the 5 -year risk of malignancy to obtain an onset of action faster than 6 weeks and approximately three-tenths of a percentage-point increase $(0.3 \%)$ in the 5-year malignancy to increase the probability that UC symptoms are under control after 1 year from $9 \%$ to $25 \%$. Respondents were also willing to accept a higher increase in 5-year risk of malignancy (above 10 tenths of a percentage point, or $1 \%$ ) to obtain an increase in the probability that UC symptoms are under control after 1 year from $9 \%$ to $50 \%$. However, this risk level lies outside of the risk range used in the DCE experimental design, as it is greater than $0.8 \%$. Therefore, we can only safely state that the maximum acceptable percent-point increase is at least $0.8 \%$ 5-year risk of malignancy.

Most of the other trade-offs included in Table 4 were not statistically significantly different from zero or were above the range of risk level included in the DCE experimental design. Physicians were also willing to accept a higher increase in 5-year risk of malignancy (above 10 tenths of a percentage point, or $1 \%$ ) to obtain an increase in the probability that UC symptoms would be under control after 1 year from $9 \%$ to $50 \%$. Physicians were also willing to accept between 4.3 and at least 8 tenths of a percentage-point increase $(0.43 \%$ and $0.85 \%)$ in the 5 -year risk of malignancy to obtain a faster onset of action.

\section{Discussion}

In this study, we elicited and compared physician and patient preferences for characteristics of UC treatments in the US. Notably, the same list of important attributes emerged from the qualitative interviews with both patients and physicians. However, preferences and conditional relative attribute importance varied considerably between the two samples. We found that patients cared far more about symptom control than the amount of time it takes for the medicine to start working. A potential explanation for this finding is that $\mathrm{UC}$ is a chronic disease, and patients who have experienced moderate to severe UC symptoms for years may prioritize durable symptom control over an earlier onset of symptom relief. For physicians, symptom control was also the most important attribute; however, when compared to the risk of malignancy, it was five times as important as the risk of malignancy, whereas for patients, the relative importance of symptom control and of risk of malignancy was similar. In addition, patients preferred all pill-dosing schedules to both subcutaneous injections and intravenous infusions, although less frequent dosing schedules were not always preferred to more frequent dosing schedules. The differences in the conditional relative importance of the treatment attributes between patients and physicians suggest that patients are thinking about the treatment features differently than their physicians and that patients are considerably more riskaverse than physicians. 


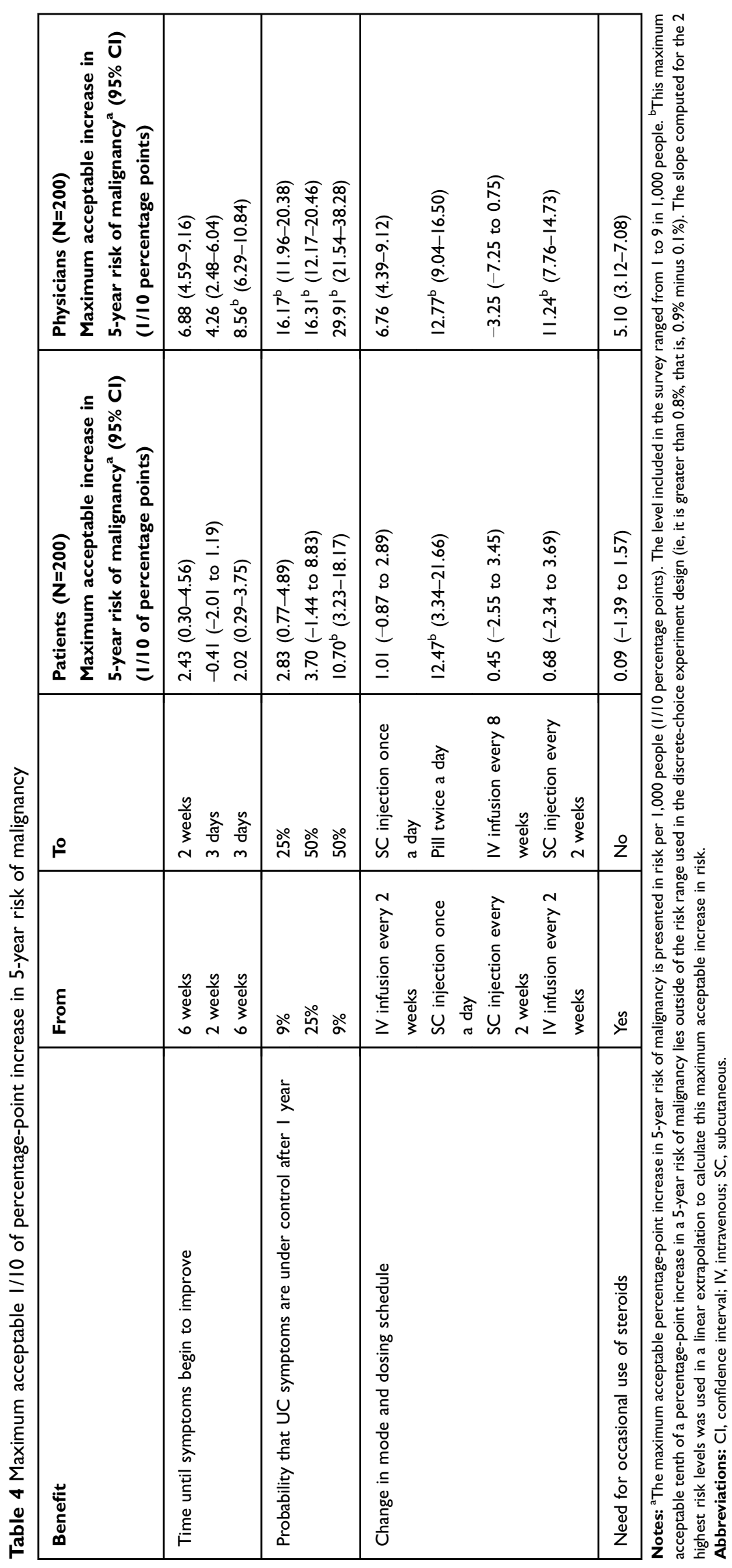


Previous studies in UC have explored patients' and physicians' preferences for medical treatment compared with surgical treatment or have evaluated patients' preferences among 5-ASA options. Hodgkins et al, ${ }^{8}$ estimated differences in patient preferences for 5-ASA treatment in mild to moderate UC in the US, United Kingdom (UK), Germany, and Canada. They found that, while clinical attributes were significantly preferred by the majority of patients, particularly in Germany, convenience attributes such as mode of administration and dosing were also important for study participants in the UK. Bewtra et $\mathrm{al}^{5}$, assessed UC patients' willingness to accept trade-offs between the risks of important medication side effects to avoid colectomy. The results from this study found that patients were willing to accept a $>5 \% 10$-year risk of dying from lymphoma or infection from medical therapy, regardless of medication efficacy, to avoid surgery. Bewtra et $\mathrm{al},{ }^{7}$ conducted a DCE among 202 patients with IBD to explore the trade-offs between varying risks of lymphoma and infection, and varying time to next IBD relapse. As in our study, the respondents in this study were willing to accept risks to achieve disease control. The authors found that patients with IBD were willing to accept an average of a $28 \%$ chance of a serious infection and an average of $1.8 \%$ chance of developing lymphoma to avoid a disease relapse over the next 5 to 10 years. Patients were willing to accept lower risks ( $11 \%$ and $0.7 \%$, respectively) for 1.5 years until the next disease relapse. Only one study, ${ }^{5}$ to our knowledge, has compared patients' and physicians' preferences for the treatment of UC. However, this study did not use a DCE approach, as its aim was not to explore preferences for features of UC medical treatments but to compare medical options with surgery. Patients, gastroenterologists, and colorectal surgeons were interviewed on different scenarios for managing acute and chronic UC. The authors found that patients (and gastroenterologists) are averse to surgery and that patient preferences for UC treatment were more aligned to those of gastroenterologists than to those of colorectal surgeons.

This study revealed that patients and physicians/clinicians have differing opinions about the relative importance of UC treatment attributes. Understanding these differences can facilitate open discussions regarding the optimal treatment approach and improve the patient treatment experience. Moreover, as shared treatment decisions vary over time and change based on patients' treatment response (or lack of response), such discussions between patients and physicians must evolve and recur. In other words, perceived risks of treatment options will change based on the benefits already obtained. Such back-andforth exchanges of incremental disease response and therapy adjustments emphasize the dynamic nature of effective "real world" shared decision making. Acknowledgement of this process is likely to improve outcomes by enabling advancing therapies and achievement of therapeutic targets, while strengthening the doctor-patient (or providerpatient) relationship. ${ }^{21}$

This study is characterized by several strengths. The study design and analyses followed best practices for DCEs, and the attributes included in the DCE were selected during in-depth qualitative interviews. The findings of these interviews are reported, as suggested by Vass et $\mathrm{al}^{22}$, in the Supplementary Data. The survey also included comprehension questions to assess whether respondents correctly interpreted attributes.

Nevertheless, some limitations of the study must be acknowledged. First, stated preferences may differ from preferences implied by actual treatment choices, and repeated choice tasks can cause cognitive fatigue and lead to measurement error. The attributes evaluated in this study represent only a subset of the factors involved in real-world treatment decisions. Other factors not included in the DCE, such as medication co-payments and deductibles, may influence decision-making. While results from online DCE surveys are often similar to face-to-face interviews, there may be some selection bias resulting from conducting the survey online using an opt-in panel. The survey respondents constituted a convenience sample drawn from an online panel, and the results are subject to potential response bias. The respondents may not be representative of the broader population of patients with moderate to severe UC, potentially limiting the generalizability of the results. Respondents' UC diagnosis, UC severity, and other clinical characteristics were self-reported and were not verified with physicians or against medical records. Finally, limited sample sizes may be insufficient to detect differences in preferences.

\section{Conclusion}

This study found that the same important treatment attributes emerged from the qualitative interviews for both patients and physicians but that preferences and relative importance among the attributes varied between the samples. Namely, patients prioritize symptom control over time to symptom improvement, a finding that is consistent with expectations, given that $\mathrm{UC}$ is a chronic disease in which patients have dealt with symptoms for several years. 
For patients, all pill-dosing schedules were more preferred to both subcutaneous injections and intravenous infusions; however, less frequent dosing schedules were not always preferred to more frequent dosing schedules. For physicians, symptom control was five times as important as the risk of malignancy, whereas the relative importance between symptom control and risk of malignancy was about the same for patients. Future research should explore whether patients' treatment preferences differ based on treatment response, disease severity, or demographic characteristics and whether physicians' treatment preferences differ by years of practice and geographic region.

Differences in the conditional relative importance of the treatment attributes between patients and physicians suggest that patients prioritize treatment features differently than physicians. There is a need for improved communication between patients and physicians about the relevant benefits and risks of treatment to improve shared decision-making for patients with UC.

\section{Abbreviation list}

5-ASA, 5-aminosalicylate; CI, confidence interval; DCE, discrete-choice experiment; GED, General Education Development; IBD, inflammatory bowel disease; IV, intravenous; RPL, random-parameters logit; SC, subcutaneous ; SD, standard deviation; SSI, Survey Sampling International; UC, ulcerative colitis; UK, United Kingdom; US, United States.

\section{Ethics approval and informed consent}

The RTI International Institutional Review Board (IRB) determined that this study meets the criteria for IRB exemption under the Code of Federal Regulations (section 45CFR46.101 (b) specifically) because the research involved the use of survey procedures, the information obtained was recorded in such a manner that human subjects could not be identified, directly or through identifiers linked to the subjects, and any disclosure of the human subjects' responses outside the research would not reasonably place the subjects at risk of criminal or civil liability or be damaging to the subjects' financial standing, employability, or reputation. In the qualitative phase, each interview participant signed and dated an informed consent form before the interview. All respondents to the DCE survey provided electronic informed consent and received compensation for time spent participating. The study complied with the Declaration of Helsinki.

\section{Data availability}

The data used in the analyses are available from the corresponding author on reasonable request.

\section{Acknowledgments}

The authors gratefully acknowledge Kimberly Moon of RTI Health Solution for overall project management for this study, Dr Allen Mangel for his help during the development of the survey instrument, and Kate Lothman of RTI Health Solutions for her help during the development of this manuscript. This study was funded by Pfizer Inc. through a contract with RTI Health Solutions. The abstract of this paper was presented at the 2018 Advances in Inflammatory Bowel Diseases (AIBD) Conference; December 13, 2018. Orlando, FL as a poster presentation. The poster and the poster's abstract are available online in the archives of Advances in Inflammatory Bowel Diseases (AIBD): https://aibd.scientificposters.com/ epsAbstractAIBD.cfm?id=1.

\section{Author contributions}

Amy Marren and Marco DiBonaventura initiated the study. Marco Boeri, Brett Hauber, Kelley Myers, and Claire Ervin designed the study, with input from Amy Marren, Marco DiBonaventura, and Joseph C Cappelleri. Marco Boeri, Brett Hauber, Kelley Myers, and Claire Ervin conducted the statistical analyses. Marco Boeri led development of the manuscript, with input from Kelley Myers, Claire Ervin, Amy Marren, Marco DiBonaventura, Joseph C Cappelleri, Brett Hauber, and David T Rubin. David T Rubin provided clinical input on the study. All authors contributed to data analysis, drafting and revising the article, gave final approval of the version to be published, and agree to be accountable for all aspects of the work.

\section{Disclosure}

Claire Ervin, Kelley Myers, Marco Boeri, and Brett Hauber are employees of RTI Health Solutions, which were paid consultants to Pfizer in connection with the development of this manuscript. Amy Marren, Macro DiBonaventura, and Joseph C Cappelleri are employees and shareholders of Pfizer. Marco Boeri and Brett Hauber received project funding from Pfizer during the conduct of the study. Dr David T Rubin reports grants and personal fees from Abbvie, Genentech/Roche, Janssen Pharmaceuticals, Prometheus Laboratories, Shire, and Takeda, personal fees from Abgenomics, Allergan, Inc., Arena Pharmaceuticals, Biomica, Bristol-Myers Squibb, 
Dizal Pharmaceuticals, Ferring Pharmaceuticals, Inc., Lilly, Merck \& Co., Inc., Medtronic, Napo Pharmaceuticals, Pfizer, and Target PharmaSolutions, Inc., outside the submitted work. The authors report no other conflicts of interest in this work.

\section{References}

1. Kappelman MD, Moore KR, Allen JK, Cook SF. Recent trends in the prevalence of Crohn's disease and ulcerative colitis in a commercially insured US population. Dig Dis Sci. 2013;58(2):519-525. doi:10.1007/ s10620-012-2371-5.

2. Mowat C, Cole A, Windsor A, et al. IBD section of the British Society of Gastroenterology. Guidelines for the management of inflammatory bowel disease in adults. Gut. 2011;60(5):571-607. doi:10.1136/ gut.2011.237727

3. NICE. Surveillance report 2017 - crohn's disease: management (2012) NICE guideline CG152 and Ulcerative colitis: management (2013) NICE guideline CG166. [Updated June 22, 2017]. Available from: https://www.nice.org.uk/guidance/cg166/resources/surveillance-report -2017-crohns-disease-management-2012-nice-guideline-cg152-andulcerative-colitis-management-2013-nice-guideline-cg 1664484819632/chapter/Surveillance-decision?tab=evidence. Accessed April 23, 2019.

4. Rubin DT, Ananthakrishnan AN, Siegel CA, et al. ACG clinical guideline: ulcerative colitis in adults. $\mathrm{Am} J$ Gastroenterol. 2019;114:384-413. doi:10.14309/ajg.0000000000000152

5. Bewtra M, Kilambi V, Fairchild AO, et al. Patient preferences for surgical versus medical therapy for ulcerative colitis. Inflamm Bowel Dis. 2014;20(1):103-114. doi:10.1097/01.MIB.0000437498.14804.50

6. Byrne CM, Tan KK, Young JM, Selby W, Solomon MJ. Patient and clinician preferences for surgical and medical treatment options in ulcerative colitis. Colorectal Dis. 2014;16(4):285-292. doi:10.1111/ codi. 12538.

7. Bewtra M, Fairchild AO, Gilroy E, et al. Inflammatory bowel disease patients' willingness to accept medication risk to avoid future disease relapse. Am J Gastroenterol. 2015;110:1675-1681. doi:10.1038/ ajg.2015.321.

8. Hodgkins P, Swinburn P, Solomon D, Yen L, Dewilde S, Lloyd A. Patient preferences for first-line oral treatment for mild-to-moderate ulcerative colitis: a discrete-choice experiment. Patient. 2012;5 (1):33-44. doi:1178-1653/12/0001-0033/\$49.95/0

9. Braun V, Clarke V. Using thematic analysis in psychology. Qual Res Psychol. 2006;3:77-101. doi:10.1191/1478088706qp063oa
10. Bridges JFP, Hauber AB, Marshall D, et al. Conjoint analysis applications in health-a checklist: a report of the ISPOR Good Research Practices for Conjoint Analysis Task Force. Value Health. 2011;14:403-413. doi:10.1016/j.jval.2010.11.013

11. Ryan M, Gerard K, Amaya-Amaya M. Using Discrete Choice Experiments to Value Health and Health Care. Dordrecht, the Netherlands: Springer; 2008.

12. de Bekker Grob EW, Ryan M, Gerard K. Discrete choice experiments in health economics: a review of the literature. Health Econ. 2010;21 (2):145-172. doi:10.1002/hec.1697

13. Clark M, Determann D, Petrou S, et al. Discrete choice experiments in health economics: a review of the literature. Pharmacoeconomics. 2014;32:883-902. doi:10.1007/s40273-014-0170-x

14. Sawtooth Software Technical Paper Series. The CBC system of choice-based conjoint analysis Version 8. Orem, UT; 2013.

15. Johnson FR, Lancsar E, Marshall D, et al. Constructing experimental designs for discrete-choice experiments: report of the ISPOR conjoint analysis experimental design good research practices task force. Value Health. 2013;16:3-13. doi:10.1016/j. jval.2012.08.2223

16. Kuhfeld W, Tobias F, Garratt M. Efficient experimental design with marketing research applications. J Mark Res. 1994;31:545-557. doi: $10.1177 / 002224379403100408$

17. Train K. Discrete Choice Methods With Simulation. 2nd ed. Cambridge, United Kingdom: Cambridge University Press; 2009.

18. Train K, Sonnier G. Mixed logit with bounded distributions of correlated partworths. In: Scarpa R, Alberini A, editors. Applications of Simulation Methods in Environmental and Resource Economics. Dordrecht, Netherlands: Springer Publisher; 2005:117-134.

19. Swait J, Louviere J. The role of the scale parameter in the estimation and comparison of multinomial logit models. J Mark Res. 1993;30 (3):305-314. doi:10.1177/002224379303000303

20. Hauber AB, González JM, Groothuis-Oudshoorn CG, et al. Statistical methods for the analysis of discrete choice experiments: a report of the ISPOR conjoint analysis experimental design task force. Value Health. 2016;19(4):300-315. doi:10.1016/j.jval.20 16.04.004

21. Rubin DT, Krugliak Cleveland N. Using a treat-to-target management strategy to improve the doctor-patient relationship in inflammatory bowel disease. Am J Gastroenterol. 2015;110(9):1252-1256. doi:10.1038/ajg.2015.86

22. Vass C, Rigby D, Payne K. The role of qualitative research methods in discrete choice experiments: a systematic review and survey of authors. Med Decis Making. 2017;37(3):298-313. doi:10.1177/ 0272989X16683934
Clinical and Experimental Gastroenterology

\section{Publish your work in this journal}

Clinical and Experimental Gastroenterology is an international, peerreviewed, open access, online journal publishing original research, reports, editorials, reviews and commentaries on all aspects of gastroenterology in the clinic and laboratory. This journal is indexed on American Chemical Society's Chemical Abstracts Service (CAS).
The manuscript management system is completely online and includes a very quick and fair peer-review system, which is all easy to use. Visit http://www.dovepress.com/testimonials.php to read real quotes from published authors. 